\title{
Agile Project Management for IT Projects in SMEs: A Framework and Success Factors
}

\author{
Daniel O'Sheedy \\ Southern Cross Business School, Southern Cross University, Australia \\ Emails: daniel.osheedy@gmail.com
}

Shankar Sankaran

School of the Build Environment, University of Technology, Sydney, Australia

Emails: Sankaran.Sankaran@uts.edu.au

\begin{abstract}
Project management methods have been developed from industry practices and international standards to ensure a higher rate of success for information technology projects. These have been widely used in large organisations effectively. However, when projects are implemented in a small or medium-sized enterprise environment, there is often a lack of an established method of project management or skilled project implementers who can use methods used in large organisations. As project workers find themselves pressured to become more responsive to business demands, it is becoming commonplace for smaller organisations to forgo formal project management practices. This is often due to the fact that small projects are viewed as simple to deploy, suffer from a lack of resources, or are given low prioritisation by the organisation. Additionally, the current project management standards are frequently perceived by SMEs as complicated and overly bureaucratic, something undesirable in regards to timeconstrained or low-budget projects. Agile development is one solution to the problem of overly complex methods that has recently been adopted in the field of software production, and has gained considerable popularity with smaller organisations. Through action research conducted in two closely-related SMEs in Austria, a final agile project management framework was produced in the end of the study. The developed framework consists of four phases of initiating phase (addressing objectives), planning phase (focusing on project backlog), executing and controlling phase (looking at iterative project cycles), and closing phase (working on project deliverables). Meanwhile some suggestions associated with the success of the developed agile project management framework are presented as well.
\end{abstract}

Keywords: Agile Method, Project Management, SMEs, IT Projects, Managing Implementation.

\section{The Environment of Participating SMEs}

The organisational structure of the businesses involved in the research environment is not entirely standard, and will be described in this section to highlight some of the research challenges and customisations that may be needed as the research progresses. Pseudonyms have been used to preserve the privacy and confidentiality of the organisations and people involved in this research.

The research took place in two SME companies in Austria, Publish-Com and Media-Com, where the researcher (the first author of the paper) held the role of IT manager in both organisations. Publish-Com is a publishing company based in Vienna, though the owner of the company has his headquarters situated in Salzburg, approximately 400 kilometres away. The owner has more of a strategic and market placement role, rather than a hands-on management role, and as such is not heavily involved in the daily operations of the company. The owner does however keep abreast of the progress of the business, through regular updates and meetings with the relevant company managers.

Initially a single company, as Publish-Com grew in size it was split into two separate companies. PublishCom, comprised of 70 employees, remained a standalone company, whereas Media-Com, the offshoot company comprised of 40 employees, became a subsidiary of the parent company, Global-Com. A 
separate London subsidiary that produced similar products was reduced in size to 20 employees, and in turn became a branch office of Media-Com. PublishCom continued to concentrate its resources on producing a weekly society and style magazine, whereas Media-Com became a media and publishing company, though its publications are focused on sport and lifestyle magazines, in addition to corporate publications. These corporate publications were usually unique publications for other subsidiary companies in the group, and included such items as marketing brochures, coffeetable books, or yearbooks detailing the highlights of sporting clubs belonging to the owner.

Due to the size of Publish-Com and Media-Com, and since both companies were operating from the same building, it was decided that job duplications would be eliminated where possible. Therefore the management, marketing and IT departments began to service both Publish-Com and Media-Com. The relevant part of the organisation chart is depicted in Figure 1, showing the researcher's role as the IT Manager for both PublishCom and Media-Com.

The researcher's line manager and chain of command in Publish-Com is the same as in Media-Com. Additionally, for the researcher there was a taskoriented line of command that needed to be observed, which is comprised of managers from Global-Com's global IT operations department in the group headquarters. Therefore, though the management approach for the two companies is similar, there are several significant differences in the management approach that is used for the IT department for PublishCom and Media-Com.

In Publish-Com the researcher had quite broad discretionary powers on how to best achieve the IT goals of the company, and projects were able to be implemented with a relatively small lead time. For the purpose of this research it was possible to modify and refine the project management processes in PublishCom with relative ease, due in part to the role that the researcher held. However in Media-Com more restrictions were in place, as more preparation for projects was required, more people were usually involved, and project teams possessed geographically dispersed members. Whereas the process style in Publish-Com was quite informal, Media-Com is part of the world-wide group of companies that make up Global-Com, and must therefore adhere to formal processes, procedures and governance practices. This made Media-Com a little less flexible in regards to the research as the major processes were already in place, though it was a relevant, to determine if what functioned in Publish-Com was also able to be implemented within the formal constraints of Media-Com.

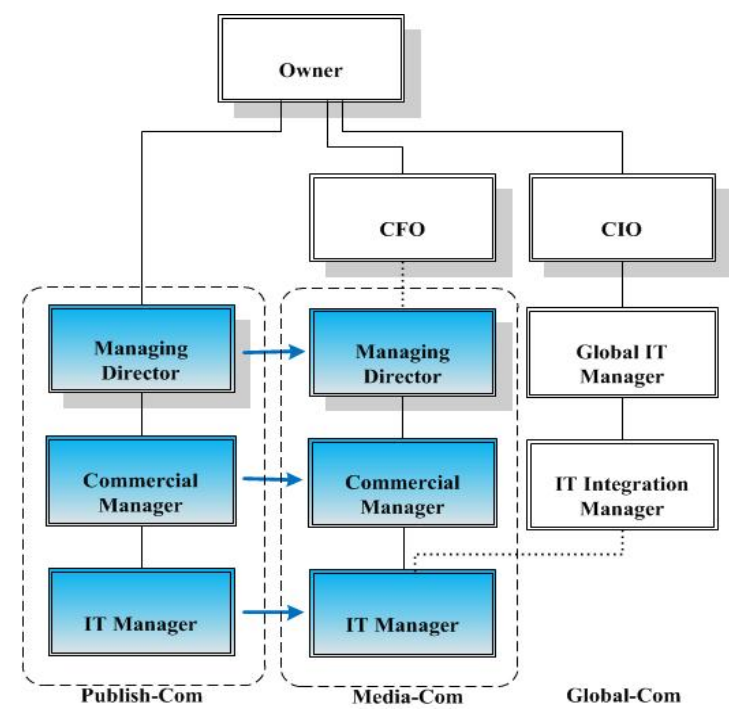

Figure 1: The relevant organisation structure for the research environment (Source: developed for this research)

As this research was conducted in Austria, we will briefly take a look at the current SME environment in this country and examine any unique characteristics and constraints which may arise from this situation. In a recent paper by Turner, Ledwidth and Kelly (2010) ${ }^{1}$ they noted that in their view, generally Austrian companies would adopt a more autocratic way of dealing with employees, when compared to other European countries. However, the results that Hofstede $(2001)^{2}$ arrived at demonstrate a slightly different viewpoint. Hofstede investigated the cultural differences between inhabitants of different countries in his work, comparing and indexing the cultural attributes of countries. Hofstede noted that Austria as a country exhibits quite a low power distance index. A low score in this index typically presents itself in the work organisation in the form of a flat organisational structure and democratic leaders. It also produces managers who rely heavily on personal experience, making decisions based on past encounters. Additionally, these types of managers were noticed to rely heavily on their subordinates, and subordinates in turn expect to be consulted on matters, such that a consultative leadership style will often produce the best results in this type of 
culture. Austria also possesses a high uncertainty avoidance index score, which measures a society's tolerance for uncertainty. As a rule, people living in a society with a higher score are more tolerant of opinions different to their own, and attempt to have as few rules as possible.

Though there are many large multi-national organisations operating in Austria, this research was undertaken in an entrepreneurial SME environment. Thus it was an ideal environment to introduce a new method of conducting projects in a team-oriented manner, as this is a style of working that would fit well into the culture of this country. The tolerance for uncertainty in the organisation also seemed higher than average, due to the type of work, and the market within which they were operating. This boded well for a positive reaction to the research, where new ideas would be introduced into the work-place, and different processes would need to be discussed.

Although the Austrian market has some unique characteristics, due to globalisation and the fact that Austria is a developed industrialised country many of the issues and findings pertaining to SMEs in other developed counties also apply to Austria ${ }^{3}$. Additionally, common company strategies and methods of doing business were comparable to organisations situated in other developed countries, establishing similarities with SMEs in those countries ${ }^{4}$. These similarities across countries enabled the use of research that had been completed in other countries to be applied to this unique situation.

\section{The Final Agile Project Management Framework}

The principle aim of this study was to investigate the topic of agile project management, which combined the foundations of traditional project management with modern agile development tools and techniques. The goal of this investigation was to discover if the concepts already in use in software development could be used in a different field, that of project management. The PMBOK was chosen as the foundation of the project management processes and procedures, and agile components primarily from Scrum and Extreme Programming were blended into a style that was applied to the research questions. The modified agile project management framework was then tested in the field, in an IT setting of two SME organisations. A final agile project management framework was produced in the end of the study (see Figure 2), it consists of four phases of initiating phase (addressing objectives), planning phase (focusing on project backlog), executing and controlling phase (looking at iterative project cycles), and closing phase (working on project deliverables).

During the second half of the research Publish-Com was acquired by Global-Com. To provide a better usage of resources, it was decided that Publish-Com would be merged with Media-Com. This merger produced a major change in the company environment, and once again it was possible to test the project management framework in a unique setting. The project therefore, was to merge Publish-Com into Media-Com, and to integrate the systems of these two companies with those of Global-Com. This work involved the internal IT team that was in charge of the systems for Publish-Com and Media-Com, the corporate IT team that was responsible for Global-Com's IT systems, and external contractors that were brought in by Global-Com to assist with the integration work.

\subsection{The initiating phase of the agile project management framework}

The first phase of the project was the initiating phase. The first task in this phase was to establish the project objectives. In a large project this would take the form of a project charter ${ }^{5}$. This is also a necessary component for the success of an agile project ${ }^{6}$, though it may be presented in a slightly different format, and will contain three components at a bare minimum. The first component is the vision, which defines why the project is being conducted. For this project, the vision was to improve the business and IT processes and systems of Publish-Com, and bring them in line with the corporate standard, in order to improve the efficiency of the organisation. The second component of the charter is the mission, which mentions the work that will be undertaken to achieve the project's vision. The mission for this project was to merge Publish-Com with MediaCom, and integrate them more closely with the corporate standard business and IT systems and practices. This would involve moving all the existing systems to new systems, and introducing standardised systems and software to the organisation.

The final important component of the charter is the success criteria. Establishing the definition of project success early on is a vital process in the project ${ }^{7}$, and it is vital that this be done in a collaborative manner with key stakeholders ${ }^{8}$. Initially, due to the complexity of the project, the IT teams had established an execution phase 
timeline of approximately two months. This was designed to ensure minimal disruption of the employees' workflow, as during this period PublishCom would still be required to produce a weekly magazine. However a new managing director was appointed around this time, and his desire was for the work to happen using a 'big bang' approach, with all systems being converted and introduced in one weekend. The technical problems with the timing were explained, and a compromise was reached with what would be delivered on the weekend, and what could be delivered at a later date. By discussing the problems openly, the different stakeholders were able to come to an agreement of what was needed for the project to be viewed as a success.

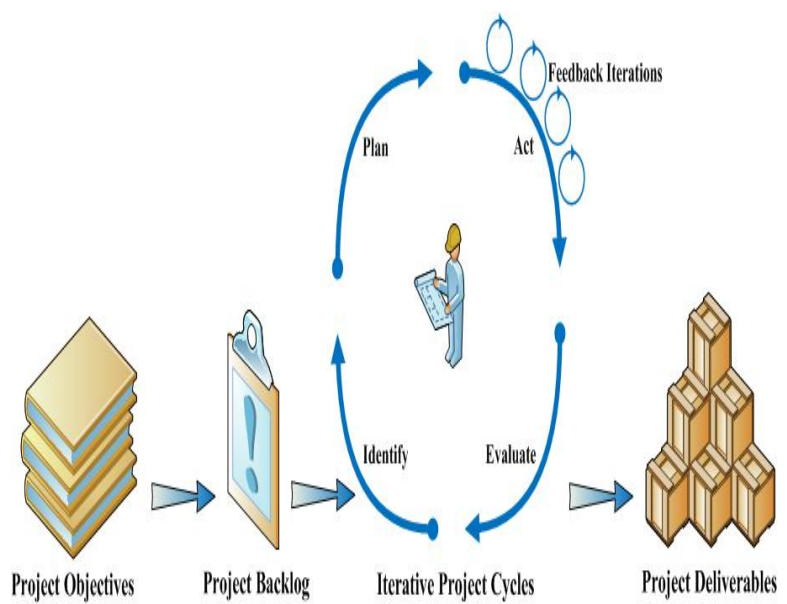

Figure 2: The final agile project management framework (Source: developed for this research)

\subsection{The planning phase of the agile project management framework}

The next phase of the project was the planning phase. Here the project was planned, and the necessary task sequencing that would be required set out. The first step in this phase was to collect the requirements for the project, which is the process whereby the stakeholders' needs are determined to bring about the objectives of the project $^{5}$. In this project there were several stakeholders, with varying requirements. The IT department of Global-Com required a unified and standardised IT environment, as an outcome of the project. The managing director wanted to merge the companies Media-Com and Publish-Com, and align the new business to the goals of Global-Com, and the staff of Media-Com and Publish-Com wanted uninterrupted access to the IT tools that would allow them to do their job properly. The project scope is then constructed using these requirements. This scope is a detailed description of the project, and lists the work that will need to happen to satisfy the project requirements ${ }^{5}$.

The next step in this process was to create a work backlog. This is a breakdown of the work that will need to happen to fulfill the project scope. The backlog can be viewed as a list of 'work chunks' that are still unfinished, with a priority assigned to each chunk. The goal is to focus on the work with the highest priority first, providing the best value for effort with each iteration $^{9}$. Some of the tasks may need to occur before others in the timeline, and there will therefore also be dependencies that need to be considered. For example, in this project the new backend servers needed to be operational before the users' computers could be configured with the new software environment. This backlog was created and monitored in specialised agile management software, using features that are found in the manual style of task board tool often found in Scrum development environments. This allowed the project management team to see the project's progress at a glance, and allow them to take the responsibility for certain tasks in the backlog.

As there were several contractors involved in this project, the WBS tool was also used to define and control the work that these contractors would undertake. This is a useful tool for the control of costs, and also removes uncertainty when project members are not local to the project team. Microsoft Project was also used to create the base timeline for the project, and this information was shared with the project shareholders. This stage is also where cost estimates are formed. Capital and operating expenditure were approved, and necessary resources organised. Agile project management, whilst taking a nimble approach to project management, still needs to provide accountability for resource usage and cost containment.

\subsection{The executing and controlling phase of the agile project management framework}

The next two stages are closely link in this framework. These two phases are the executing and controlling phases, where the project work is conducted, and there are constant checks in place to make sure the proper work is being conducted. As noted earlier, the basis for 
the project execution has been taken from traditional action research, and consists of the four stages, Identify, Plan, Act, and Evaluate ${ }^{10}$.

The project backlog was used as the input for each execution cycle. A chunk of work from the backlog is taken by a project team, and the end-goal for that chunk is identified. The work required to achieve that goal is determined, and a plan of action is formulated. The project team then puts the plan into action, in the act section of this phase. This is the part of the project where the implementation work takes place, and may take days or weeks. During the act section the team that is working on that chunk will meet regularly, to discuss the progress and ensure that the project is heading in the proper direction. These meetings ensure that team remains focussed on the cycle's output goal, and allows for minor adjustments as changes occur in the project environment. These feedback iterations are important to the smooth adjustment of the project work to changing requirements.

Depending on the amount of effort involved in completing a chunk of work, a cycle may take days or weeks, and the timing of the feedback iterations need to be adjusted accordingly. Once a chunk of work has been completed, the result is evaluated. The chunk of work must be completed to specification, and also fit into the project objective. If there is a deviation in the project work from the project objectives, an unforeseen problem has presented itself during the project, or a change in the business during the project execution has occurred, then this can feed back into the project objectives and project backlog, depending on the size and scope of the changes needed. These work cycles may occur serially, or even simultaneously, if multiple teams are working on different parts of the overall project. For example, with the merger project, two teams were working on different aspects of the transition to the new mail server. The local team was working on upgrading the client facing software, and the remote team from Global-Com was working on the back-end configuration. These two teams, whilst working on separate chunks, were working towards the same end objective.

Changes can be implemented quickly when using this style. During the act section of one of the earlier cycles, it was discovered that the corporate standard software was not compatible with the new Macintosh operating system. A fix was procured from the vendor, a new image created, and the implementation continued. During the final weekend there were multiple teams working on multiple chunks, all with goal of completion by the end of the weekend. However a vital server suffered a catastrophic failure on the first morning. This immediately affected a majority of the other tasks in progress, as it was the central point for authentication and authorisation. The server team started to work on this problem immediately, and the other teams gathered to determine the best course of action for the other tasks. The teams were able to find several workarounds, and were able to continue the project work, albeit at a slower pace. As this final weekend involved the finishing stages of several complex tasks, the feedback iterations were carried out every two or three hours, as the work was complex and progressing quickly. This allowed the teams to continually reprioritise work chunks, get updates on the progress of other teams, and optimise the work flow for the next few hours. These meetings were very brief, with teams giving succinct status reports and proposed plans for the next few hours. Despite the major problems that occurred during the weekend, the project teams were able to achieve the main objectives with only a few low priority tasks left incomplete.

The key feature on the executing and controlling phase is that there are multiple feedback iterations in the act section. This allows the project team to critically assess two aspects of the ongoing project implementation work. The first aspect is whether the project work is working towards the project's objectives. If the team finds that their efforts are heading off in a tangent, then they can reconcentrate their efforts back towards the goal. The second aspect is whether the environment in which the project is occurring has changed significantly. If the environment has changed, so that the project goal is no longer fully relevant, then the project work will need to be adjusted to a more relevant goal. This can apply especially in a project that is dealing with an emerging product, or in a research-based environment. For example, if an organisation is introducing a new product to a fast moving market, as insights about the market become clearer assumptions made in the early stages of the project may no longer be fully accurate, and adjustments to the project will need to be made in order to introduce those improved insights. Agile differs from many traditional methods of project management, in 
that changes are embraced, even late in the project cycle. Changes to the project environment need to be address quickly, though the project manager needs to remember that these changes do not come without a cost. The project manager will need to communicate the change in costs to the stakeholders, as a significant change can increase the labour or time requirements significantly.

\subsection{The closing phase of the agile project management framework}

The last phase of the project is the closing phase. Here the project team compares the project objectives once again with the delivered work. This makes sure that the project that was promised has been delivered, and has been accepted by the stakeholders. If there are any unforeseen results of the project, or other issues, than these need to be addressed and rectified. The project team also needs to document the lessons learnt from the project, so that the knowledge gained in this endeavour is stored for future project teams to benefit from.

\subsection{A Summary of the components of the agile project management framework}

There are many well-established tools and procedures that were used during this project, though the use of each particular tool needs to be assessed for each project. The project manager will need to determine which tools are necessary and will add value to the project management process. The tools and procedures used in this project have been summarised in Table 1, which has been divided into the separate project phases.

This is only a brief and summarised description of the project process, used to provide a bit more detail into the agile framework, and should not be viewed as a cookbook on how to implement projects. Only the processes that were featured foremost in the spotlight for this research were discussed, though there were other processes that occurred and must also be taken into consideration. This may include such processes as determining the budget for the project, human resource planning, and procurement, to mention but a few. The project manager still needs to take these types of processes into consideration, and the PMBOK (PMI 2008) and is an excellent basis upon which to start.

As described in the previous chapter, this framework was tested over a period of several months by using it for the management of multiple projects. During this time, it was shown that the framework proved beneficial for the management of IT projects. One of the benefits was that it helped to ease the introduction of project requirement changes, even in advanced stages of implementation. It also provided a set of processes for managing projects in an SME environment that was flexible and non-bureaucratic, whilst still providing adequate processes to guide and control the projects.

Table 1: Tools and procedures used during the example project (Source: developed for this research)

\begin{tabular}{|l|l|}
\hline Project Phase & $\begin{array}{l}\text { Tools and procedures } \\
\text { implemented }\end{array}$ \\
\hline Initiating & Project charter \\
\hline Planning & $\begin{array}{l}\text { Project scope } \\
\text { Project work backlog } \\
\text { Agile development task board } \\
\text { Work breakdown structures } \\
\text { Gantt charts }\end{array}$ \\
\hline Executing & $\begin{array}{l}\text { Agile development task board } \\
\text { Agile development update } \\
\text { meetings }\end{array}$ \\
\hline $\begin{array}{l}\text { Monitoring \& } \\
\text { Controlling }\end{array}$ & Burn down charts \\
\hline Closing & Knowledge base of lessons learnt \\
\hline
\end{tabular}

Returning the focus to the primary research question, the agile framework help with the successful completion of projects, as the framework helped the project team to respond quickly to changes as they arose, and also to manage the complexities of a project in a changing environment. A selection of the projects involved very unique situations, where customised products and solutions were being developed for niche markets. Therefore project lead times were often very brief, and it was common for major changes to be introduced at short notice. The agile project management framework helped improve the flow and the management of projects, especially in situations where there was a high degree of uncertainty and not all factors were initially known.

The framework presented here should not be considered a step-by-step process manual, prescribing a 'correct' path to manage projects. Rather, it should be viewed as a flexible project management system, which is adaptable and responsive to environment changes. The framework is still in the developmental stages, and further research needs to be undertaken into the model itself. This will determine if this tool can be used in a wider range of situations and produce favourable results, and whether it is also a scalable solution, with potential uses outside of an SME environment. Additionally, extra study needs to be focused on whether it is possible to design a toolkit so that benefits can be reaped, not only by trained and professional 
project managers, but also by the average project worker. This is particularly relevant, as the current framework requires advanced training or knowledge on the part of the project manager.

\section{Recommendations for the Agile Project Management Framework}

Meanwhile some suggestions associated with the success of the developed agile project management framework include:

- $\quad$ There are real benefits to be gained from implementing an agile project management style

- $\quad$ Agile project management needs the support of the business owners, especially in an SME environment

- $\quad$ Agile project management is better suited to smaller environments

- $\quad$ Agile is not a panacea, and needs to be used as a tool in a suitable setting

- $\quad$ The agile project management framework must be adjusted to the company structure and culture to work most efficiently

- The framework must be customised, incorporating elements of both agile and PMBOK

- $\quad$ Small teams work best in an agile environment, though teams can scale for larger projects by adding extra teams

- $\quad$ To become a skilled project manager requires many hours of experience and training

- $\quad$ The project manager's management style needs to be matched to the project, and they need to be trained in this role.

\subsection{There are real benefits to be gained from implementing an agile project management style}

The agile project management framework designed during the course of this research was proven to be successful in an SME environment. Different levels of processes can be applied to a project when implementing an agile project management method. It is possible to change the level of processes that are applied to a project, as the agile project management framework is very adaptable. Therefore for small and non-complex projects, the processes can be reduced in the amount and complexity, whereas for more complex projects, the full weight of more advanced PMBOK and agile processes can be applied to. The adaptability and versatility of this framework was a valuable factor in the management of projects during this research, and assisted in the successful completion of these projects.

\subsection{Agile project management needs the support of the business owners, especially in an SME environment}

Agile project management diverges from the standard methods of project management in several areas. Due to the innovative nature of agile project management, if the business owner does not fully support the introduction of project management processes, then there is a higher potential for acceptance problems within the organisation. The acceptance problems usually arise because employees may not perceive the benefit or the need for using such a system.

Even though a business owner may not actively oppose a system, if they do not fully support it then it can fail due to a lack of support. Additionally, as project management will often require additional resources to implement, if senior management in a parent company do not provide these necessary resources, or if some parts of the company actively oppose the introduction of new processes, then the project manager will encounter difficulties in introducing the proper procedures and processes to implement such a management style.

\subsection{Agile project management is better suited to smaller environments}

In this study it was noted that an agile project management framework works best for small and medium projects in an SME environment. It was also noted that as projects grew in complexity the framework began to demonstrate several deficiencies. For example, tracking large and complicated projects can be more difficult for agile project management, as well as coordinating work schedules when there are a large number of project workers involved. This is a demonstration of the scalability concerns of this style of project management, and the more complex a project becomes the more issues arise when project changes are introduced. It has also been noted that traditionally larger teams are more adverse to change, and agile methods may need to be introduced to these environments with more conservative goals at first. 


\subsection{Agile is not a panacea, and needs to be used as a tool in a suitable setting}

Although agile project management is a useful tool, it cannot solve all problems, and is not suitable for every situation. One problem that project managers encounter is that functional managers have many priorities that compete for their attention. Unless the project manager is a skilled communicator, then requests for project resources can easily be ignored or relegated to a secondary position by these functional managers.

It has also been noted that historically managers and business leaders have generally shown little interest in business research, and that these managers will not necessarily adopt method that have been proven in research studies. However, there is still a real avenue open that the demand for improved methods will come from the project management practitioners themselves, rather than being implemented as a strategic management choice for the organisation.

\subsection{The agile project management framework must be adjusted to the company structure and culture to work most efficiently}

The agile project management framework is by design flexible and adaptable. However, this framework should not be applied in the same form to each organisation in the same manner. Rather, it should be adjusted to the structure and the culture of the organisation, to gain the biggest impact. For example, an organisation with a 'female mindset' will usually distinguish itself in the form of a flatter company structure with less bureaucracy, where leaders often making more intuitive decisions. The agile project management style works well in the type of a company which displays this characteristic female mindset. This is primarily due to a reduction in the amount of rigid rules and bureaucracy in this style of company, which are detrimental to agile project management. If the company culture is rigid and bureaucratic, then this style of project management will usually be more difficult to introduce.

\subsection{The framework must be customized, incorporating elements of both agile and PMBOK}

As a component of the agile framework, the PMBOK is also a valuable project management framework, but there is a need for the framework to be customised, including 'enough' of them for successful project management. The level of detail required for the project management processes depends on the level of complexity within a project. A project that is simple in its scope and execution will not need the same controls and processes that are required for a project that is more complex or difficult to execute. As the agile project management framework is adaptable, the level of processes that are applied to the project can be readily adapted. Therefore, for small and non-complex projects the controlling processes can be simple and limited. For more complex and difficult projects however, a wider range of $\mathrm{PMBOK}$ and agile process may need to be applied to the management tasks.

\subsection{Small teams work best in an agile environment, though teams can scale for larger projects by adding extra teams}

The delivery of projects in an SME environment primarily occurs through the efforts of small teams. It has been noted that small organisations predominantly undertake small projects, due to the amount of resources available to them. Since the project teams are smaller, the team members may be required to perform the tasks of more than a single role. For example, the project manager may also be in charge of implementation, and a project team member may be involved in the implementation of several stages of the project. A team of four members was found to be a useful size, as this enabled the project team to remain adaptable and flexible, though still allowing room to scale for slightly larger projects, with the addition of extra teams as required. The importance of coherence in the team is also noted, as the project is not successfully completed through the efforts of one or two people alone, but on the skills and efforts of the whole team. If the project team has an average of high quality in its team members, then it is most likely possible to achieve a greater level of project success than with just one or two highly trained project team members.

\subsection{To become a skilled project manager requires many hours of experience and training}

Project management is specialised field, where not all managers are equal in their abilities. One thing that differentiates between a seasoned project manager and a junior project coordinator is experience, which is gained over years of practice. It has been demonstrated that to 
reach the level of an expert in their field, a practitioner will need in excess of 10,000 hours of practice and experience. They are then able to make decisions based on these many hours of practice, often so that these decisions may appear spontaneous and arrive seemingly from what can appear to be a 'gut feeling'. However this demonstration of an expert level of knowledge should not be mistaken for pure intuition, as it is a result of prolonged training, where decisions become ingrained and a part of their everyday actions. Additionally, this expert knowledge is gained through a combination of hands-on experience and formal learning.

\subsection{The project manager's management style needs to be matched to the project, and they need to be trained in this role}

The project manager needs to possess the proper project management training for the best possibility of project success. Additionally though, the personality and management style of the project manager need to match with the style of project, otherwise the success rate will undoubtedly be affected when implementing an agile style of project management. In addition, the project manager needs the skills and personality that enable them to investigate the traditional project management tools, and put them into practice in a balanced manner with the agile development processes. Through these processes they are able to determine the best course of action for that particular project, and are able to deliver a customised project management strategy for each unique project environment. In addition to possessing broad experience in project management, the project manager will need domain knowledge as well, as domain experts are better equipped to make decisions in environments that are less structured, which is a common situation when implementing agile methods.

\section{References}

1. J.R. Turner, A. Ledwith, and J. Kelly, Project management in small to medium-sized enterprises: matching processes to the nature of the firm, International Journal of Project Management, 28 (8) (2010) 744-755.

2. G. Hofstede, Culture's consequences: comparing values, behaviors, institutions, and organizations across nations, 2nd edn. (Sage Publications, 2001).

3. M. Fink, R., Harms, and S. Kraus, Cooperative internationalization of SMEs: self-commitment as a success factor for international entrepreneurship, European Management Journal, 26 (6) (20808) 429-440.
4. K-H. Leitner, and S. Güldenberg, Generic strategies and firm performance in SMEs: a longitudinal study of Austrian SMEs, Small Business Economics, 35 (2) (2009) 169-189.

5. PMI, A Guide to the Project Management Body of Knowledge (PMBOK ${ }^{\circledR}$ guide), 4th edn. (Project Management Institute, 2008, 459).

6. G.L. Chin, Agile project management: how to succeed in the face of changing project requirements (AMACOM, 2003, 224).

7. J.R. Turner, Five necessary conditions for project success, International Journal of Project Management, 22 (5) (2004) 349-350.

8. J.R. Turner and R. Müller, Communication and cooperation on projects between the project owner as principal and the project manager as agent, European Management Journal, 22 (3) (2004) 327-336.

9. K. Schwaber, Agile project management with Scrum (Microsoft Press, 2004).

10. C. Argyris, R. Putnam, and D. McLain Smith, Action research: concepts, methods, and skills for research and intervention (Josey-Bass 1985). 